\title{
Archéopages
}

Archéopages

Archéologie et société

$40 \mid 04-07 / 2014$

Villages

\section{Villoe - Villages du haut Moyen Âge en plaine du Languedoc oriental. Maillage, morphologie et économie}

Odile Maufras, Mathieu Ott, Claude Raynaud, Marie Rochette, Liliane Tarrou, Agnès Bergeret, Marilyne Bovagne et Richard Pellé

\section{(2) OpenEdition}

Journals

Édition électronique

URL : https://journals.openedition.org/archeopages/620

DOI : $10.4000 /$ archeopages.620

ISSN : 2269-9872

Éditeur

INRAP - Institut national de recherches archéologiques préventives

Édition imprimée

Date de publication : 1 juin 2015

Pagination : 92-103

ISSN : $1622-8545$

\section{Référence électronique}

Odile Maufras, Mathieu Ott, Claude Raynaud, Marie Rochette, Liliane Tarrou, Agnès Bergeret, Marilyne Bovagne et Richard Pellé, « Villæ - Villages du haut Moyen Âge en plaine du Languedoc oriental. Maillage, morphologie et économie », Archéopages [En ligne], 40 | 04-07/2014, mis en ligne le 01 juillet 2016, consulté le 21 janvier 2022. URL : http://journals.openedition.org/archeopages/620 ; DOI https://doi.org/10.4000/archeopages.620 


\section{Villæ - Villages du haut Moyen Âge en plaine du Languedoc oriental Maillage, morphologie et économie}

Odile Maufras Inrap, UMR 514 \& «Archéologie des sciences méditerranéennes»

Mathieu Ott ${ }^{\text {Irrap }}$

Claude Raynaud CNRS, UMR 5140 «Archéologie des sciences méditerranéennes»

Marie Rochette Irrap, UMR 5140 «Archéologie des sciences méditerranéennes»

Liliane Tarrou Inrap

Avec la collaboration de Benjamin Thomas, Séverine Baudin, Quentin Guérin, Maxime Guillaume, Eric Yebdri Inrap Agnès Bergeret, Marilyne Bovagne Inrap, UMR 5140 «Archéologie des sciences méditerranéennes»

Richard Pellé Inrap, IRAA - USS 3155

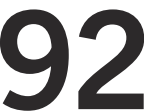

Le village de plaine en Languedoc oriental au premier Moyen Âge était encore, ces dernières années, un grand inconnu. Non qu'il fut rare entre les $\mathrm{V}^{\mathrm{e}}$ et XII ${ }^{\mathrm{e}}$ siècles - c'est au contraire manifestement la forme d'habitat la plus répandue à partir du $\mathrm{IX}^{\mathrm{e}}$ siècle -, mais parce qu'il n'a laissé que peu de traces, tant dans la documentation écrite et iconographique qu'au sol. Peu d'historiens se sont attachés au sujet, ce que la parcimonie des sources explique en grande partie. Les archéologues, de leur côté, ont peiné à en exhumer les vestiges: le village du haut Moyen Âge est resté discret ou largement inaccessible. En plaine, il est principalement construit en matériaux périssables, avec des toitures de végétaux et des murs de terre crue qui ont pourri ou fondu après l'abandon et ne sont plus détectables. Ainsi, il ne subsiste le plus souvent que les infrastructures les plus profondes: soubassement des églises, puits, silos et tombes. La découverte de groupements de telles structures au moment des diagnostics a permis de reconnaitre une vingtaine de sites d'habitat aggloméré de plaine depuis les années 1980, mais les rares maisons conservées - une seule à Saint-Jean-d'Aureilhan (Béziers, Jandot et al., 1999) et à Dassargues (Lunel, Garnier et al., 1995); aucune autour de l'église Saint-Sylvestre de Teilhan à Aimargues par exemple (Piskorz et al. 2002) - ne livrent pas de données sur l'extension, la densité ni les formes du bâti.

La répartition des villages est en revanche mieux documentée, grâce aux sources fiscales, principalement les cartulaires des abbayes et des évêchés. Les biens y sont généralement localisés dans le territoire de la cité en premier lieu (sinon dans une région géographique, plaine ou vallée), puis dans l'espace d'une villa. Le terme recouvre tout à la fois la subdivision territoriale de perception des taxes, le terroir et l'habitat rural qui y prennent place (Maufras, Mercier, 2012). La nature des biens énumérés dans les chartes des $\mathrm{IX}^{\mathrm{e}}$-XII ${ }^{\mathrm{e}}$ siècles indique d'une part le caractère rural de l'occupation (terres, vignes, jardins, vergers) et d'autre part la concentration de l'habitat en un point du terroir. En effet, les unités d'exploitation (manses) y paraissent rarement isolées sur des terres, mais confrontant des parcelles bâties, voire l'église elle-même, toujours associée à l'habitat groupé, ou son cimetière (Maufras, à paraître). Villa est ainsi, alors, synonyme de village. La confrontation avec les données archéologiques disponibles, notamment sur la villa nîmoise de Codols, suggérait jusqu'en 2011 que le groupement de l'habitat puisse avoir présenté un tissu assez lâche avec des maisons et des bâtiments d'exploitation espacés, séparés par des cours et des jardins, tels les villages du haut Moyen Âge d'Île-de-France et de Rhône-Alpes (Pomarèdes, Maufras, 2012 ; Gentili, 2010 ; FaureBoucharlat, 2001). Depuis 2011, la mise au jour et surtout la fouille sur des surfaces significatives des villae bien conservées d'Ugnac à Pennautier (Aude), de La Sabatière à Trèbes (Aude), de Roux à Castries (Hérault) et de Missignac à Aimargues (Gard) nuancent ce schéma. L'habitat groupé peut être assez dense sur certains sites, certaines parties du village - autour de l'église notamment - ou à certains moments de son occupation.

À ces découvertes récentes s'ajoutent deux sites d'habitat dont on ignore s'ils furent ou non des villae (Lallemand à Mauguio, Hérault, et Saint-Pastour à Vergèze, Gard, [ill. 1]) ainsi que les villages fouillés sur des surfaces très restreintes et ceux mal conservés, mais révélés par leurs grandes aires d'ensilage (en particulier Saint-Jean-d'Aureilhan dans l'Hérault). Tous renouvellent considérablement la documentation. 
1. Les villæcarolingiennes connues par les textes ou les prospections en basse vallée du Vidourle et du Vistre. Les sites sont implantés sur des terres largement mises en valeur depuis l'âge du Fer et exploitées semble-t-il sans interruption depuis le $\mathrm{I}^{\text {er }}$ siècle de notre ère.

L'emplacement et la forme des habitats y évoluent en revanche, de même sans doute que le faire-valoir des sols. Quatre de ces ville ont fait l'objet de diagnostics ou de fouilles archéologiques qui en ont révélé l'église et le cimetière (Missignac,

Teilhan), l'habitat

(Missignac) et les quartiers d'ensilage (Missignac,

Saint-Pastour).

2. Les villa carolingiennes connues par les textes ou les fouilles en haute vallée du Vistre. Leur répartition est régulière de part et d'autre du fleuve et le maillage se resserre au nord, entre la voie Domitienne et Marguerittes, secteur où les sources et les petits rus sont abondants. Trois villane sont pas

localisées sur la carte:

Marceglage, Taurèse et Vols.

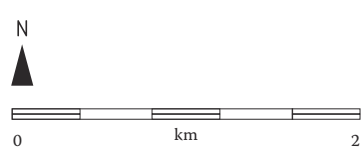

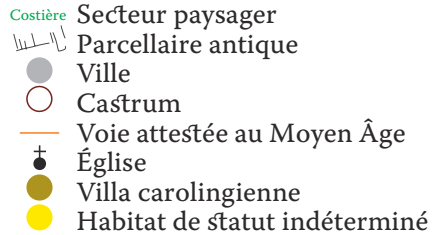

Secteur paysager

Ville

oie attestée au Moyen Âge

Habitat de statut indéterminé

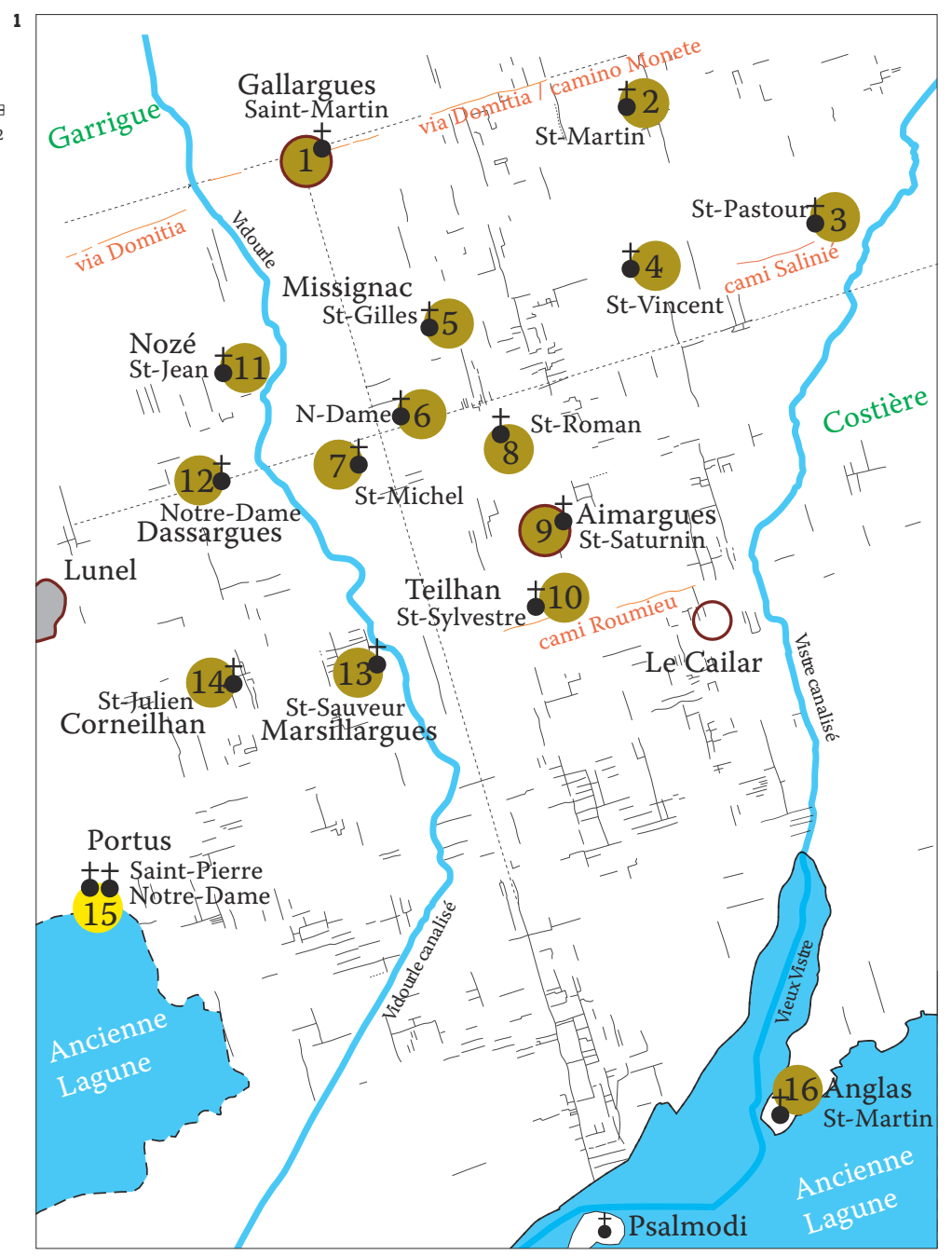

志

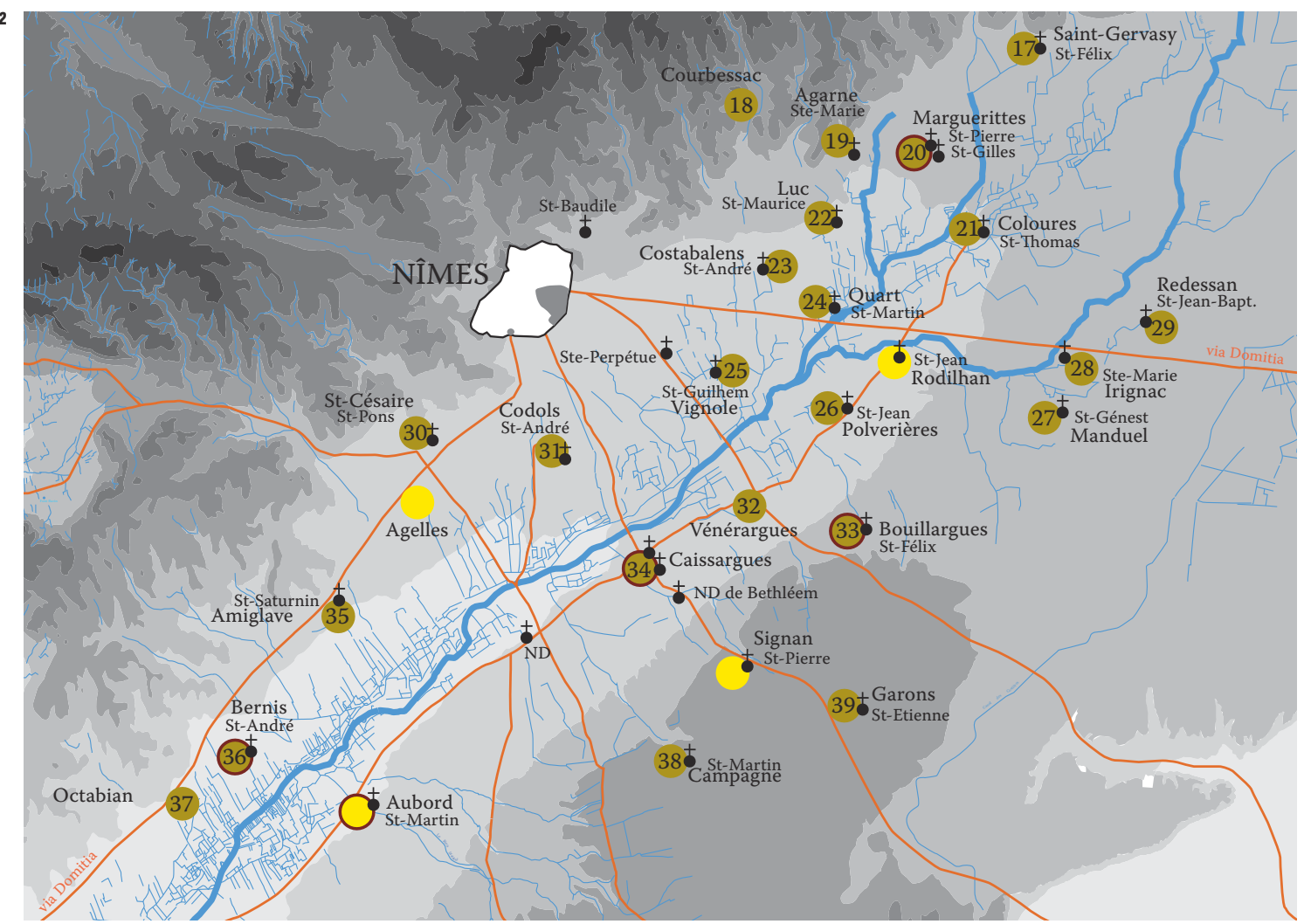



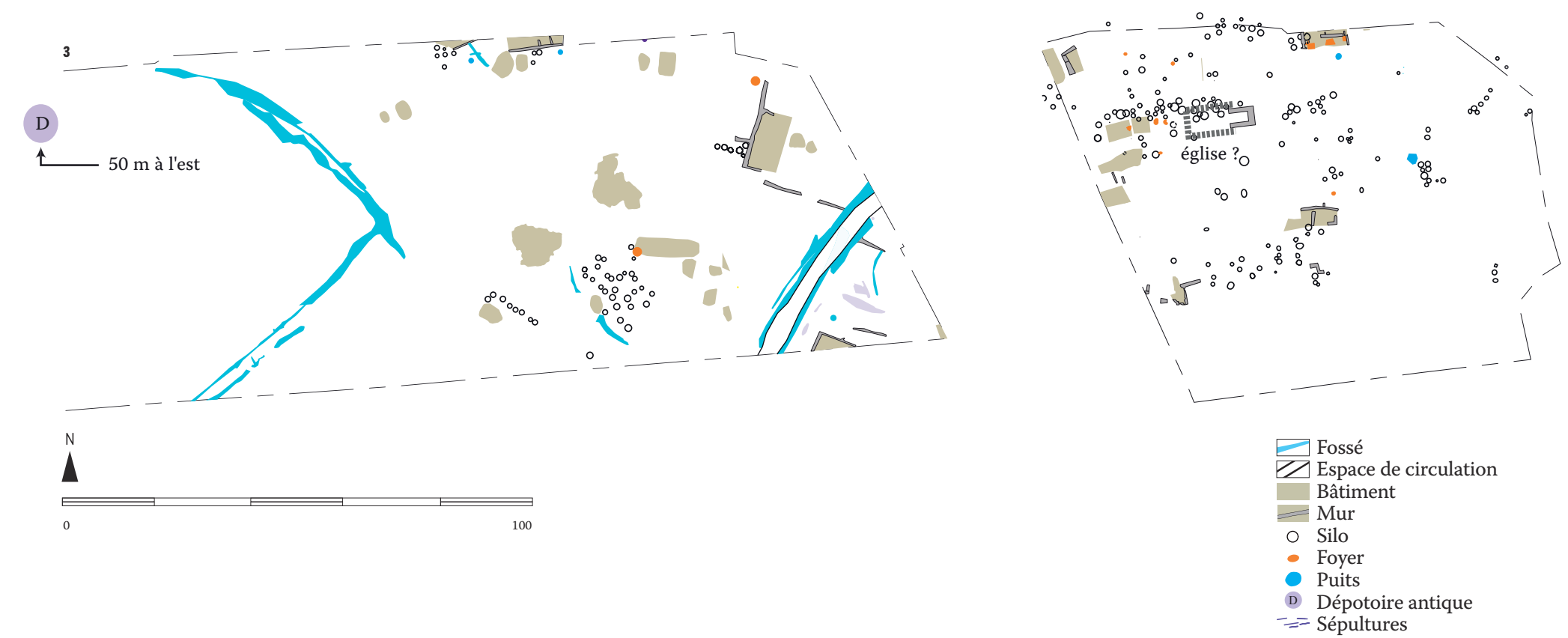

Aux questions anciennes sur la morphogenèse du village et son évolution topographique, sur le rôle de l'église dans sa formation, sur l'absence ou non de fortification avant le $\mathrm{XI}^{\mathrm{e}}$ siècle, sur la nature des constructions, sur le nombre des habitants et au-delà sur la taille du terroir, les spécificités économiques et les formes du paysage, l'archéologie est en train d'apporter de très nombreux éléments de réponse. Les données collectées sur les sites sont en cours d'étude et il n'est pas encore temps de dresser le portrait du village languedocien de plaine du haut Moyen Âge avec toutes ses variantes, mais d'ores et déjà quelques aspects peuvent être évoqués. Nous aborderons à grands traits les cas les mieux documentés ou les plus originaux, que nos recherches dans les années à venir viendront préciser.

\section{De la répartition des villages en plaine}

$\mathrm{Du} \mathrm{IX}^{\mathrm{e}}$ au XII ${ }^{\mathrm{e}}$ siècle, les villae forment dans les plaines languedociennes un maillage dense, occupant tout l'espace agricole jusqu'aux marges de la garrigue ou des palus lagunaires. Cette densité s'affirme dans la vallée du Vistre, au sud-est de la ville de Nîmes, autant que dans la basse vallée du Vidourle, aux abords de la lagune littorale. Ces deux espaces emblématiques font l'objet de recherches particulièrement poussées, mais le peuplement ne s'y distingue guère d'autres zones voisines, qu'il s'agisse de la plaine autour de l'ancien évêché de Maguelone (au sud de Montpellier), d'un bassin de garrigue comme la Vaunage à l'est de Nîmes, ou encore de la moyenne vallée de l'Hérault étudiée par Laurent Schneider.

Avec des établissements de taille et de morphologie comparables, implantés à 1 ou $2 \mathrm{~km}$ de distance, les deux zones test présentent des densités voisines: 26 villae au sud de Nîmes, soit un site pour $0,95 \mathrm{~km}^{2}$, 14. villae en bas Vidourle, soit un site pour $0,71 \mathrm{~km}^{2}$ [ill. 1 et 2]. L'implantation des établissements semble principalement influencée par la topographie et la qualité des sols: particulièrement forte aux abords des zones alluviales des cours d'eau littoraux, où certaines villae se trouvent à moins de $1 \mathrm{~km}$ de leurs voisines, la densité faiblit dès que l'on aborde les pentes de la garrigue, et s'annule dans les espaces deltaïques, fortement palustres au Moyen Âge. La basse vallée du Vidourle illustre cette répulsion des marges: au sud des villae de Teilhan et Corneilhan [ill. 1, $n^{\circ} 10$ et 14], on ne trouve plus que l'établissement de Saint-Martin d'Anglas sur une avancée de la terrasse des Costières bordée d'étangs et le Portus, agglomération portuaire dotée de deux églises et échappant au statut de villa.

Ce maillage opportuniste n'apparaît guère influencé par un tropisme urbain, connu dans l'Antiquité : ni agrégation ni glacis centrifuge ne s'observent au voisinage de Nîmes; en haute Vistrenque comme en Vaunage voisine, le maillage ne se distingue pas des eśpaces plus éloignés. Partout semblent primer la qualité et l'accessibilité des ressources, comme le montre l'exemple du Vidourle à proximité duquel se pressent les établissements parmi les plus vastes, avec la plus forte densité entre Saint-Jean de Nozé, Saint-Gilles de Missignac et Saint-Saturnin de Nodels. Sur ces secteurs, la proximité des villae ne semble pas résulter d'une fragmentation de l'habitat, bien au contraire.

Après une apparente stabilité durant plus de trois siècles, durant lesquels on n'enregistre guère de désertion ni de création, le maillage des villae connaît une vague d'abandons au cours du XIII ${ }^{\mathrm{e}}$ siècle. Progressivement, la moitié à deux tiers des établissements disparaissent de la carte. Ni massif ni synchrone, cet abandon connait d'amples décalages d'un site à l'autre; précoce ou rapide ici, plus tardif ou progressif ailleurs, le phénomène ne semble pas attribuable à une cause unique de type épidémique ou guerrier. En réalité, les textes 


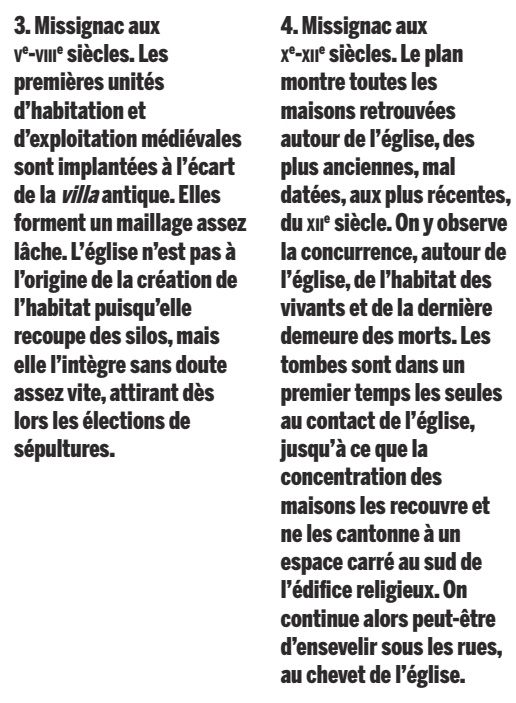

4. Missignac aux

$\chi^{e}-{ }^{e}{ }^{e}{ }^{e}$ siècles. Le plan montre toutes les autour de l'église, des plus anciennes, mal datées, aux plus récentes, du xII ${ }^{e}$ siècle. On y observe la concurrence, autour de l'église, de l'habitat des vivants et de la dernière demeure des morts. Les premier temps les seules au contact de l'église, jusqu'à ce que la maisons les recouvre et ne les cantonne à un space carré au sud de d'ensevelir sous les rues, au chevet de l'église. comme l'archéologie soulignent un processus de concentration du peuplement, qualifié d'incastellamento à partir du cas des campagnes $\mathrm{du}$ Latium, et connu, à des titres divers et selon des rythmes propres, dans nombre de régions européennes. En Languedoc oriental, le castrum d'initiative seigneuriale naît aux alentours de l'an mil. Longtemps resté modeste, il étend son emprise sur la société et l'économie aux XII ${ }^{\mathrm{e}}-\mathrm{XIII}^{\mathrm{e}}$ siècles, créant une dynamique centripète qui absorbe progressivement l'essentiel de la population et vide les anciennes villae. En Vidourlenque comme dans le nîmois, les castra du Caylar, d'Aimargues, de Gallargues, Lunel, Marguerittes, Manduel, Bouillargues, Caissargues, Garons, Milhau, Bernis et Aubord absorbent, chacun, deux à six anciennes villae [ill. 1 et 2] dont ne subsistent bientôt que des masures et quelques équipements agricoles autour des églises restées au centre de bénéfices ecclésiastiques, coquilles vides qui se maintiendront, pour cette raison fiscale, jusqu'à la Révolution. Un compoix du XIV ${ }^{\mathrm{e}}$ siècle (registre d'imposition), conservé à Lunel, offre une image éclairante du processus : les terroirs des anciennes villae n'apparaissent plus que comme sections du finage agricole. L'évolution de la villa de Missignac illustre bien ce phénomène. Concentré autour de l'église au plus tard au $\mathrm{X}^{\mathrm{e}}$ siècle, son habitat atteint sa plus grande densité aux $\mathrm{XI}^{\mathrm{e}}$ et XII ${ }^{\mathrm{e}}$ siècles avant d'être progressivement déserté entre la fin du XII ${ }^{\mathrm{e}}$ siècle et les premières décennies du XIII ${ }^{\mathrm{e}}$ siècle. Dans le deuxième quart du XIII ${ }^{\mathrm{e}}$ siècle n'en subsistent semble-t-il que l'église dont la façade a cependant été démontée, le cimetière qui accueille de nouvelles tombes encore quelque temps et quelques silos au quartier de stockage. La population a certainement migré vers les villae qui ont survécu, celles qui se sont fortifiées et sont devenues des castra, principalement Gallargues au nord-ouest ( $n^{\circ} 27$ ), Aimargues au sud ( $n^{\circ} 35$ ) et Aigues-Vives au nordest (hors cadre), si l'on en croit l'extension de leurs terroirs actuels qui couvrent l'ancien finage de Missignac et se rejoignent tous les trois quelques mètres au nord de son église.

Le terme d'inurbamento conviendrait mieux encore pour décrire le processus, car celui-ci ne se limite pas à la croissance des castra mais englobe certaines villae, certes minoritaires, qui se maintiennent, deviennent de gros villages et absorbent des villae voisines, à l'exemple de Lunel-Viel qui a survécu à la concurrence du castrum voisin de Lunel.

\section{L'origine et la topographie du village médiéval}

Les villae médiévales ne semblent pas être, dans la majorité des cas documentés, des formations ex nihilo, même si elles ne se superposent pas toutes exactement à un site d'habitat antérieur.

En haute Vistrenque, où le développement actuel de la ville de Nîmes a occasionné de nombreuses enquêtes archéologiques, l'habitat rural du HautEmpire montre une densité importante et une répartition entre des établissements domaniaux imposants - les villae gallo-romaines - et des établissements plus modestes dont certains fonctionnent en satellites des précédents. Un seul exemple de superposition d'une villa du Moyen Âge à une villa antique est attesté: Codols, avec son occupation continue et son église implantée à quelques dizaines de mètres du centre domanial 

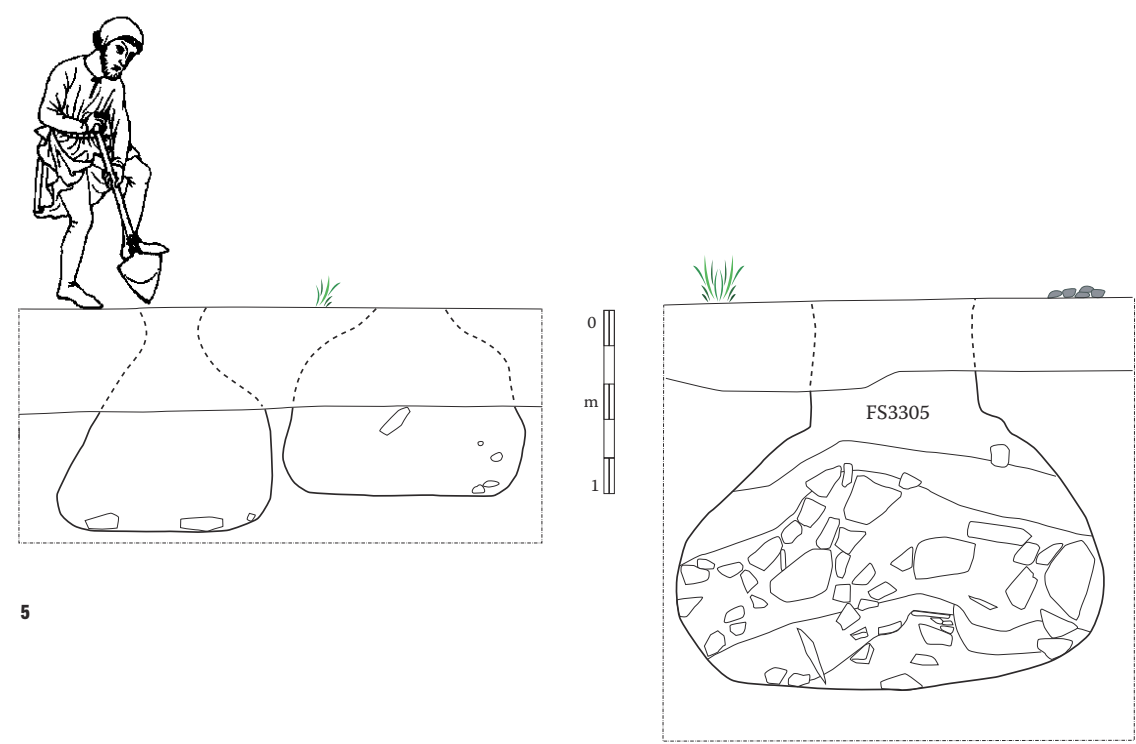

5. Un des trois très grands silos de l'aire de

Missignac : FS3305. Avec sa capacité de

5350 litres, soit 6 à 8 fois plus que les silos

ordinaires du site, il

répond à une fonction

spécifique qu'il est

difficile d'élucider. II a pu

contenir le produit de la

dîme, ou bien la semence

pour un lot de parcelles

vaste et collectif, ou une

réserve commune pour

pourvoir aux besoins des

villageois en cas de

mauvaise récolte, voire

autre chose encore.

6. Le village de Roux au

début du xill' siècle. Du

manse carolingien au

village, l'établissement

achève alors sa mutation.

En 1280, il est mentionné

parmi les in villes et

forteresses 11

dépendantes de la

baronnie de Castries.

Deux générations plus

tard, il semble déserté.

7. Le mur d'enceinte de

Roux, vu ici depuis l'ouest.

est conservé sur $1 \mathrm{~m}$

d'élévation en moyenne.

Flanqué d'un fossé et

d'une levée de terre, il

englobe, au nord de

l'emprise, un tronçon de

la voie Domitienne. Sous

les arbres à l'arrière-plan

coule le Bérange.

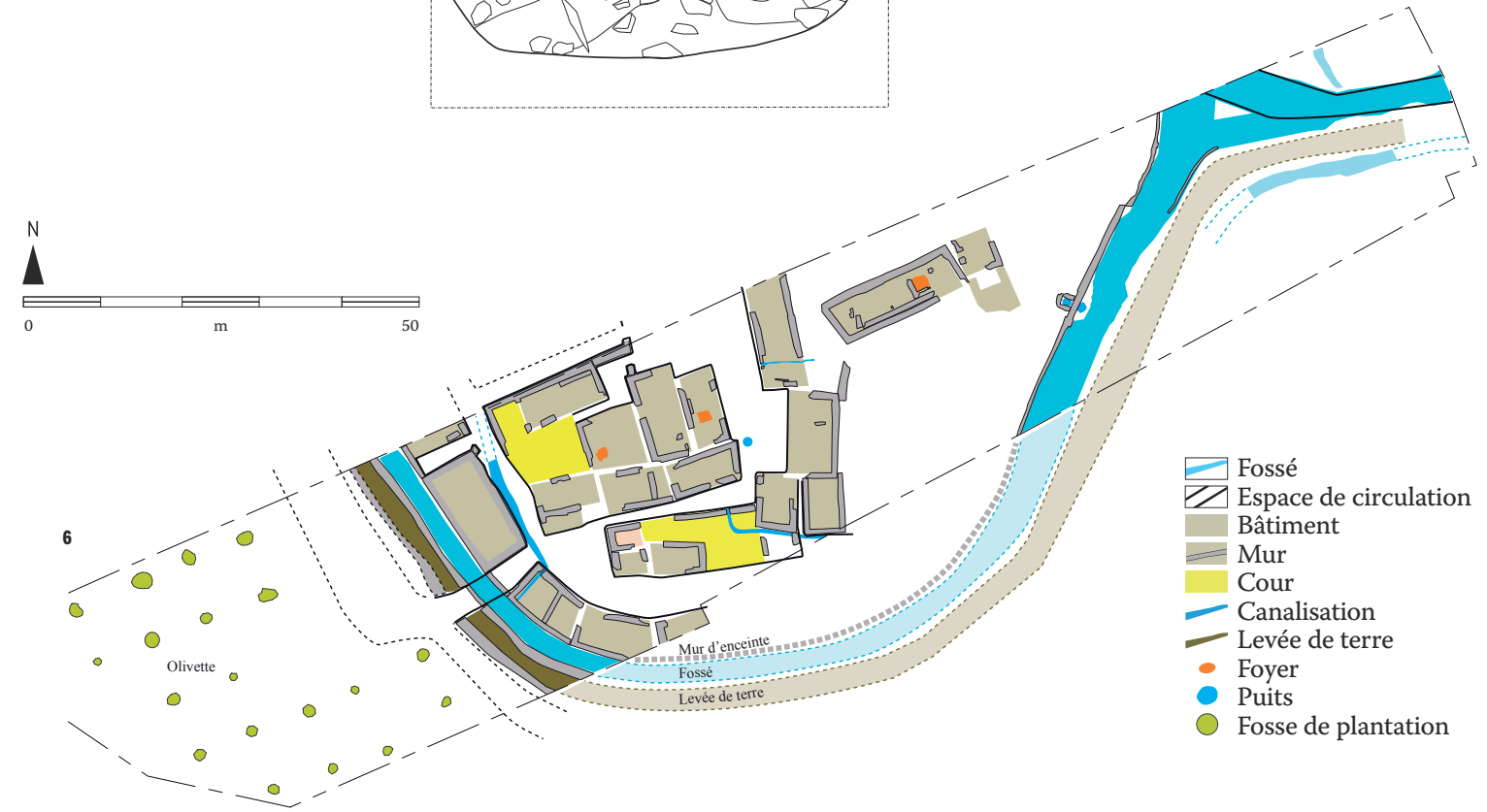

7

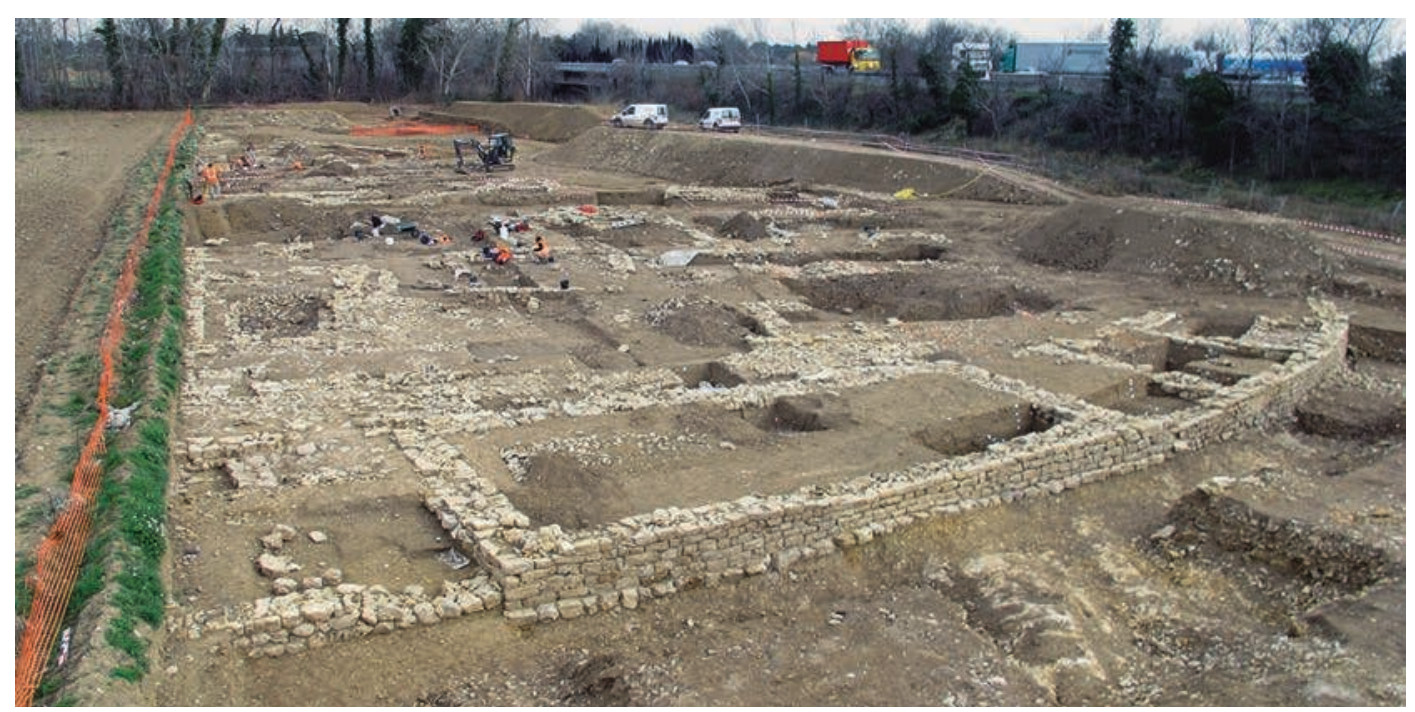


du Bas-Empire (Pomarèdes et al., 2012). À Milhau, la villa antique est en bordure du lit majeur du Vistre. Elle est abandonnée au $\mathrm{V}^{\mathrm{e}}$ siècle et devient, dès le $\mathrm{VI}^{\mathrm{e}}$ siècle, un quartier dévolu à l'ensilage, lui-même en forte expansion à partir du $\mathrm{IX}^{\mathrm{e}}$ siècle, tandis que l'habitat se décale de quelques centaines de mètres vers la hauteur où il s'agglomère (Maufras, à paraître). Trois autres sites sont dans ce cas où le village médiéval se déplace à une très faible distance (Agelles, Caissargues et Octabian) et, dans deux cas au moins, il succède à un établissement antique modeste (à Saint-Cézaire et Signan). Cette continuité tient à la permanence de l'occupation rurale de ce secteur qui est périurbain depuis la Protohistoire. Ici, point de grande vague de déprise agraire ni de reconquête agricole par les défrichements : les terroirs paraissent stables entre Antiquité et Moyen Âge. Ailleurs en Languedoc oriental, les cas de villa antiques abandonnées au $\mathrm{II}^{\mathrm{e}}$ et surtout au $\mathrm{III}^{\mathrm{e}}$ siècle abondent. Cependant la permanence de l'occupation jusqu'au $\mathrm{VII}^{\mathrm{e}}$ ou $\mathrm{IX}^{\mathrm{e}}$ siècle est aussi fréquemment attestée, notamment chaque fois que l'érosion des sites n'a pas tronqué trop bas les vestiges. En témoignent les cas emblématiques de la Gramière (Gard, Buffat, 2005) ou au-delà, en rive gauche du Rhône, de Sallians (Drôme, Le Roy et al., 2011). Nous n'excluons pas cependant l'existence de formation de villages médiévaux ex nihilo en Vistrenque et nous supposons qu'ils puissent même être nombreux dans les secteurs de l'arrière-pays où les crises démographiques ont été plus marquées et les désertions de sites antiques plus durables.

Si les textes permettent de comptabiliser et cartographier de nombreuses villae carolingiennes, très peu en revanche ont fait l'objet de fouilles archéologiques et les formes de transition entre la villa antique du $\mathrm{V}^{\mathrm{e}}$ siècle et la villa médiévale $\mathrm{du} \mathrm{IX}^{\mathrm{e}}$ siècle restent largement méconnues. Quelques sites toutefois livrent des indices qui vont dans le sens de l'éclatement du centre domanial antique en plusieurs unités d'habitation et d'exploitation, sur le site même de la villa antique d'abord, puis autour de l'église ensuite. Codols à Nîmes donne un exemple de l'amorce du processus : à la charnière des $\mathrm{IV}^{\mathrm{e}}$ et $\mathrm{V}^{\mathrm{e}}$ siècles, la villa domaniale est monumentalisée en même temps que se construit, en mitoyenneté, une modeste ferme, probable première étape d'une fragmentation de la propriété ou plus probablement du faire-valoir (Pomarèdes et al., 2012). À Missignac en Vidourlenque, l'emplacement de la villa antique n'est pas connu, mais on la restitue à proximité d'un dépotoir du Haut-Empire, $300 \mathrm{~m}$ à l'ouest de l'église Saint-Gilles [ill. 3]. Au v siècle, un petit établissement équipé de caves, de silos et d'un puits est installé entre le dépotoir antique et la future église. Il est occupé un laps de temps mal déterminé. Nous ignorons s'il est isolé ou contemporain des quelques autres unités les plus anciennes du site, l'une abandonnée $\mathrm{au}$ VIII $^{\mathrm{e}}$ siècle, les autres non datées, et si le site est déjà doté d'un lieu de culte chrétien. À Codols et à Costebalens, le vocable de l'église - Saint-André - suggère une fondation précoce, aux $\mathrm{V}^{\mathrm{e}}-\mathrm{VI}^{\mathrm{e}}$ siècles comme nombre de créations de la Gaule méridionale (Schneider, 2014). Les chapelles Saint-Vincent de Soulages (Saint-Maurice Navacelles, Hérault) et Saint-Martin-de-Castries (La Vacquerie, Hérault) ont leurs vestiges les plus anciens datés respectivement des $\mathrm{VI}^{\mathrm{e}}-\mathrm{VII}^{\mathrm{e}}$ et du VIII ${ }^{\mathrm{e}}$ siècle. À Missignac, l'église n'est peut-être pas antérieure $\mathrm{au} \mathrm{X}^{\mathrm{e}}$ siècle, mais dès sa création elle attire l'implantation concurrente des maisons et des tombes jusqu'à un point de saturation qui conduit à la création d'un cimetière restreint et clos [ill. 4]. C'est au IX ${ }^{\mathrm{e}}$ siècle que les récentes découvertes archéologiques placent le groupement effectif des vivants et des morts autour de l'église, phénomène qui peut toutefois avoir été amorcé plus tôt.

Avec l'église, les maisons et le cimetière, les aires d'ensilage à plusieurs milliers de structures constituent les lieux communs des villae carolingiennes du Languedoc oriental. L'enceinte fossoyée ou bâtie est plus rare et ne se généralise qu'au XII ${ }^{\mathrm{e}}$ siècle dans ceux des villages qui s' « enchâtèlent ».

\section{Des aménagements partagés}

Pour l'archéologue, le village voit le jour avec le groupement des habitations et des aménagements de production en un point du terroir. Pour l'historien, le village naît lorsque ses habitants utilisent en commun des biens ou des services (Bourin, Durand, 1984). Dès lors, en particulier sur les sites qui ne conservent pas de bâti et sur lesquels les formes de l'habitat ne peuvent être définies, les aménagements et les objets susceptibles d'être collectifs peuvent servir à identifier le village, voire à en dater l'apparition. S'imposent en premier lieu des équipements communs - l'église, le cimetière et, immédiatement contre le village, l'aire d'ensilage mais aussi les rues, les places, les puits, le moulin, les fortifications.

En Languedoc oriental et en Roussillon, les aires d'ensilage couvrent de vastes quartiers d'un à plusieurs hectares, généralement situés contre le village. Elles accueillent des silos par centaines pour les plus petites et par milliers pour les autres (Schneider, 2006). Ceux-ci sont répartis par groupes (aire de Missignac) ou concentrés sur des parcelles quadrangulaires attenantes les unes aux autres (Ugnac, Saint-Pastour). Dans l'aire de Saint-Pastour, les 663 silos fouillés sont rassemblés sur des parcelles rectangulaires délimitées par des chemins. Ces groupes évoquent des propriétés ou des dévolutions particulières au sein de l'ensemble. Le rassemblement des stocks en des lieux distincts de l'habitat et en un point unique pour l'ensemble du village suggère cependant une organisation collective de l'aire: entretien du secteur et surveillance des récoltes devaient être partagés. Quelques découvertes laissent à penser que le partage pouvait s'étendre à une partie des récoltes : à Missignac, de rares très grands silos ont été aménagés pour des stocks particuliers, peut-être des fonds collectifs [ill. 5]. 

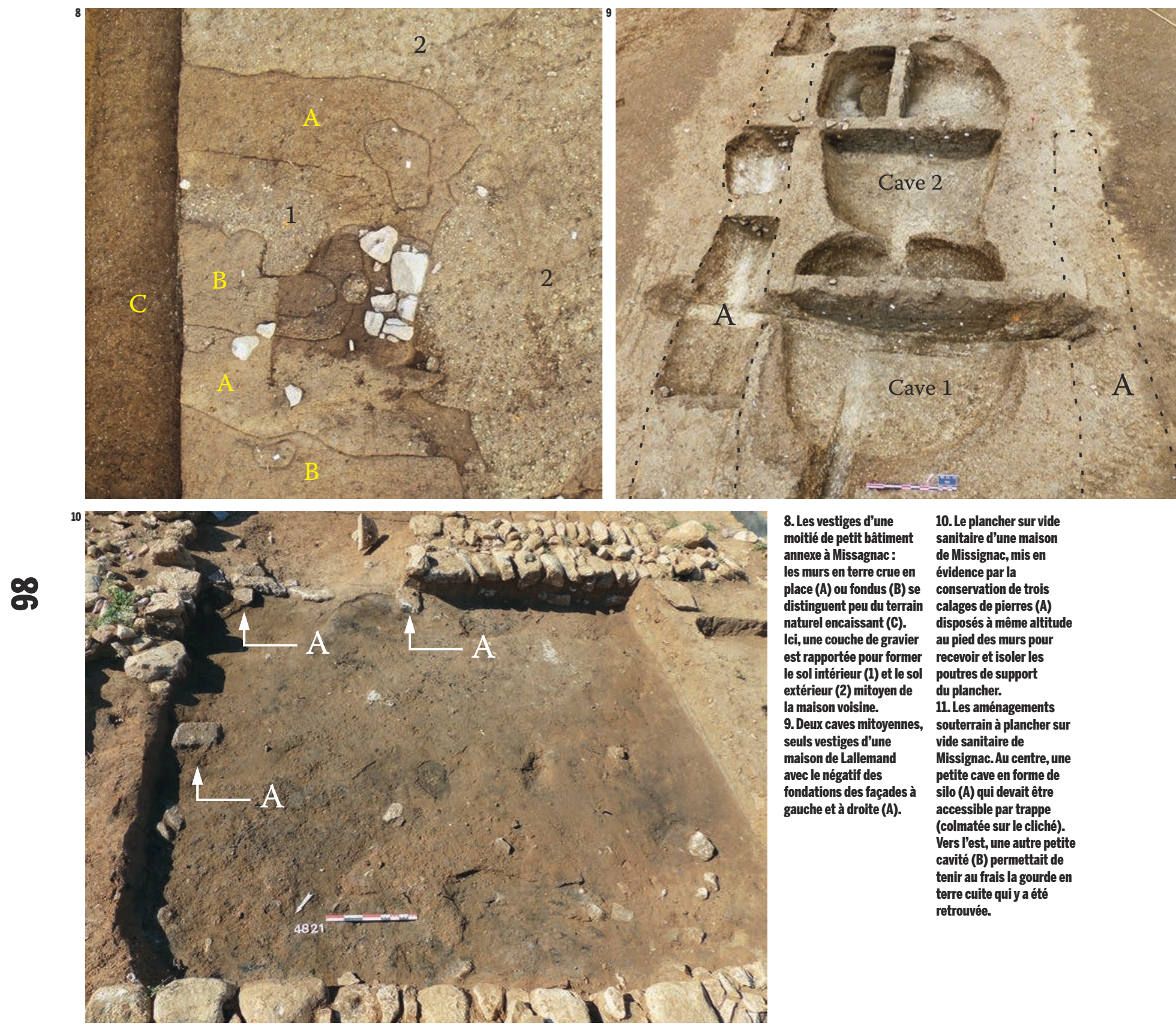

8. Les vestiges d'une

10. Le plancher sur vide moitié de petit bâtiment annexe à Missagnac:

sanitaire d'une maison

place (A) ou fondus (B) se conservation de trois

distinguent peu du terrain calages de pierres (A)

naturel encaissant (C). disposés à même altitude

Ici, une couche de gravier au pied des murs pour

est rapportée pour former recevoir et isoler les

le sol intérieur (1) et le sol poutres de support

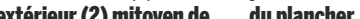

la maison voisine.

du plancher.
11. Les aménagements

9. Deux caves mitoyennes, souterrain à plancher sur

seuls vestiges d'une

maison de Lallemand

avec le négatif des

vide sanitaire de

fondations des façades

gauche et à droite (A).

petite cave en forme de

(A) qui devait être

accessible par trappe

(colmatée sur le cliché).

Vers l'est, une autre petite

cavité (B) permettait de

tenir au frais la gourde en

terre cuite qui y a été

retrouveé.

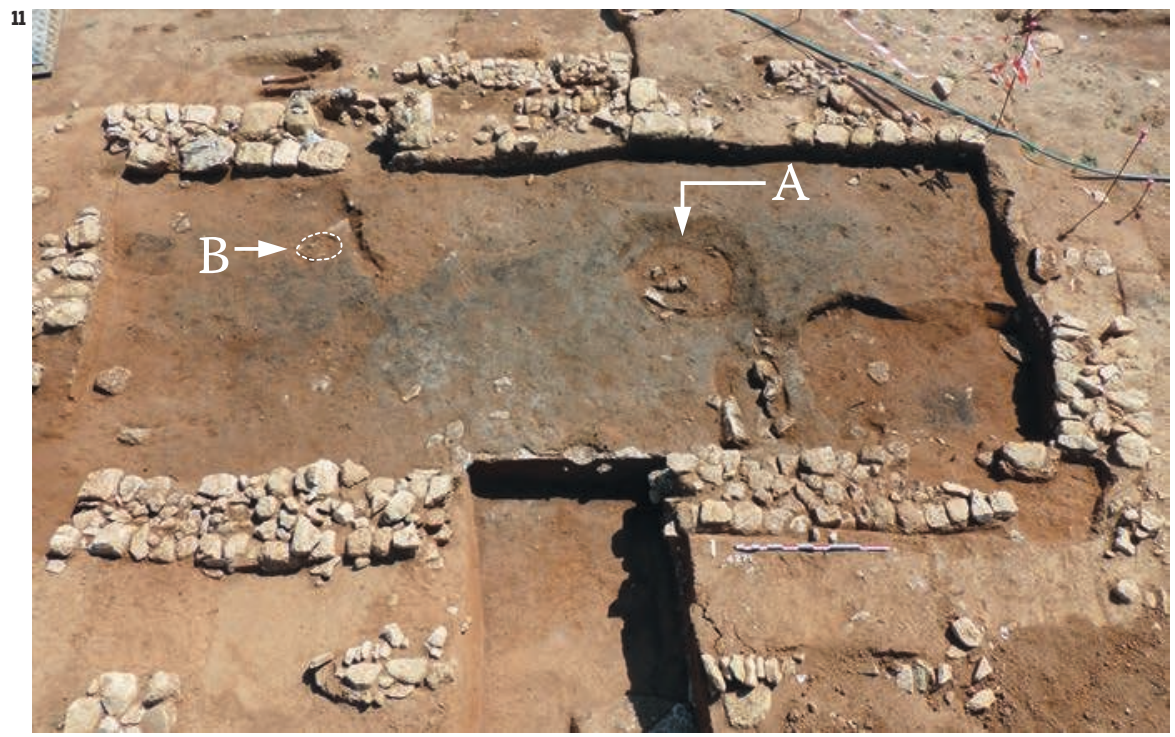


En aval du stockage, la communauté est dotée de moyens de transformation des produits agricoles. Sur l'ensemble des établissements mentionnés, la plupart des meules mises au jour sont des objets domestiques d'une quarantaine de centimètres de large, d'un poids modéré, et qu'un enfant pouvait actionner seul. Parfois, cependant, un objet diffère. C'est ainsi le cas de la grande meule de Dassargues (Lunel, Hérault), datée des $\mathrm{X}^{\mathrm{e}}$-XI $\mathrm{I}^{\mathrm{e}}$ siècles (Granier et al., 1995, p. 44). Son diamètre atteint $1 \mathrm{~m}$. Elle n'a pas été retrouvée en place, mais réutilisée en bouchon de silo. De même le site de Missignac a livré une meule tournante de $90 \mathrm{~cm}$ de diamètre qui constituait un équipement immobilier et n'occupait sans doute pas une maison, mais un espace dévolu à la mouture. L'objet a lui aussi eu un second usage: il a été retaillé pour servir de couverture à l'une des dernières tombes du site. Ces deux meules ne sont actionnables que par une ou deux personnes robustes, voire plus vraisemblablement par un animal. Cela suggère l'existence d'un moulin collectif sur chacun de ces deux sites, construit par plusieurs familles qui s'en partageaient les frais et l'usage, voire tout le village. Sur la commune de Castries, la fouille du village de Roux ( $\mathrm{IX}^{\mathrm{e}}-\mathrm{XIV}^{\mathrm{e}}$ siècle) a livré les vestiges d'un moulin hydraulique daté de la première moitié du XII ${ }^{\mathrm{e}}$ siècle. Bien que l'établissement soit situé sur la rive occidentale d'un petit fleuve côtier, le Bérange, son moulin n'occupe pas la bordure du cours d'eau. Il est localisé au cœur de l'habitat, le long d'un bief et mû par des lâchers d'eau à partir de retenues aménagées sur un ancien fossé barré par des martelières en pierre. Aménagement des martelières, curage des retenues d'eau, entretien du bief et du coursier, etc.; il est difficilement envisageable que de tels investissements aient pu être consentis par une seule famille.

Sur les sites qui restent occupés après le XII ${ }^{\mathrm{e}}$ siècle, la fortification devient, avec l'église, le signe manifeste du regroupement villageois. Vers 1150, l'établissement de Roux se voit ainsi doté d'un nouveau système défensif qui condamne l'ancien moulin [ill. 6]. Reprenant le cours d'une enceinte fossoyée héritée de la période carolingienne, une triple ligne de défense est érigée - levée de terre, fossé et mur d'enceinte - dont le périmètre avoisine $400 \mathrm{~m}$. À Roux comme dans un certain nombre de castra languedociens, le mur d'enceinte est constitué, sur son flanc occidental, de la juxtaposition des murs gouttereaux de maisons particulières [ill. 7]. Toutes sont érigées au cours du même chantier, de manière coordonnée, et répondent sans doute à un projet global. En témoignent les éléments collectifs de défense (porte, franchissement du fossé) qui s'insèrent dans le schéma directeur. Si la fortification du village répond à une nécessité commune, la réalisation du projet repose ainsi sur les propriétaires de chaque lot. Une certaine latitude leur est laissée pour l'approvisionnement en pierres ou pour la mise en œuvre des matériaux qui diffère quelque peu d'une maison à l'autre. Se pose évidemment la question de la maîtrise d'ouvrage: la mise en défense de Roux procède-t-elle d'une décision seigneuriale, ou d'un acte communautaire?

Intra-muros, la nouvelle trame juxtapose espaces privés - les maisons, les cours - et espaces publics. Rues et places sont soigneusement entretenues et munies de systèmes d'évacuation des eaux. Ces lieux sont en outre dotés d'infrastructures communautaires comme le puits. De même, à Missignac, les deux fours culinaires reconnus dans le village sont situés sur des espaces publics. Tout cela suppose un partage de l'investissement sans doute, et manifestement aussi un partage de l'usage pour la cuisson du pain.

Au-delà des témoins archéologiques, le sentiment communautaire trouvait sans doute racine dans certaines tâches ou travaux dont on peut penser qu'ils étaient partagés ou collectifs: la conduite et la garde des troupeaux par exemple, mais aussi la moisson, les vendanges... Pour répondre à ces questions, la documentation manque encore.

\section{La maison de village}

Dans les habitats récemment fouillés du Mas de Roux, Lallemand, Missignac, La Sabatière et Ugnac, les maisons sont conservées en fondation et très peu en élévation. Elles présentent un plan simple, de forme rectangulaire, d'une à deux pièces en général, parfois davantage, au maximum quatre à Missignac. Leur superficie varie de 25 à $100 \mathrm{~m}^{2}$, mais la plupart mesurent autour de $50 \mathrm{~m}^{2}$.

Dans la construction de ces maisons, les matériaux périssables dominent, mêlant la terre, les végétaux et le bois. La terre crue apparaît comme le matériau privilégié des élévations, mais pas seulement: à Missignac, par exemple, elle est aussi le principal matériau des fondations. Sur ce site, les murs en terre banchée reposent sur des solins de pierre, plus souvent sur des solins de terre gravillonneuse compactée et fréquemment sur des banquettes rupestres réservées au moment du creusement du vide sanitaire. Dans certaines maisons, seul le seuil est en pierre [ill. 8].

La pierre est d'usage plus fréquent en marge de la plaine, dès que l'on gagne les piémonts. Elle est systématiquement mise en œuvre en fondation des murs d'Ugnac au $\mathrm{x}^{\mathrm{e}}$ siècle et de La Sabatière au pied de la montagne Noire. Son emploi est attesté dans les élévations du XII ${ }^{\mathrm{e}}$ siècle d'Ugnac et du Mas de Roux (celui-ci au pied des garrigues de Montpellier). Sur ce dernier site, trois bâtiments conservent quelques assises d'élévation en moellons de calcaire liés à la terre sur des murs parementés et chaînés aux angles [ill. 7]. À Lallemand, il ne reste de l'habitation, en terre, que deux caves et, de part et d'autre, le négatif de la fondation des façades dont les matériaux - solins de bois ou plus probablement de pierre ont disparu [ill. 9].

Les maisons ne sont pas toutes couvertes de tuiles, tant s'en faut si l'on en croit la faible part de ce matériau dans les niveaux de démolition. 


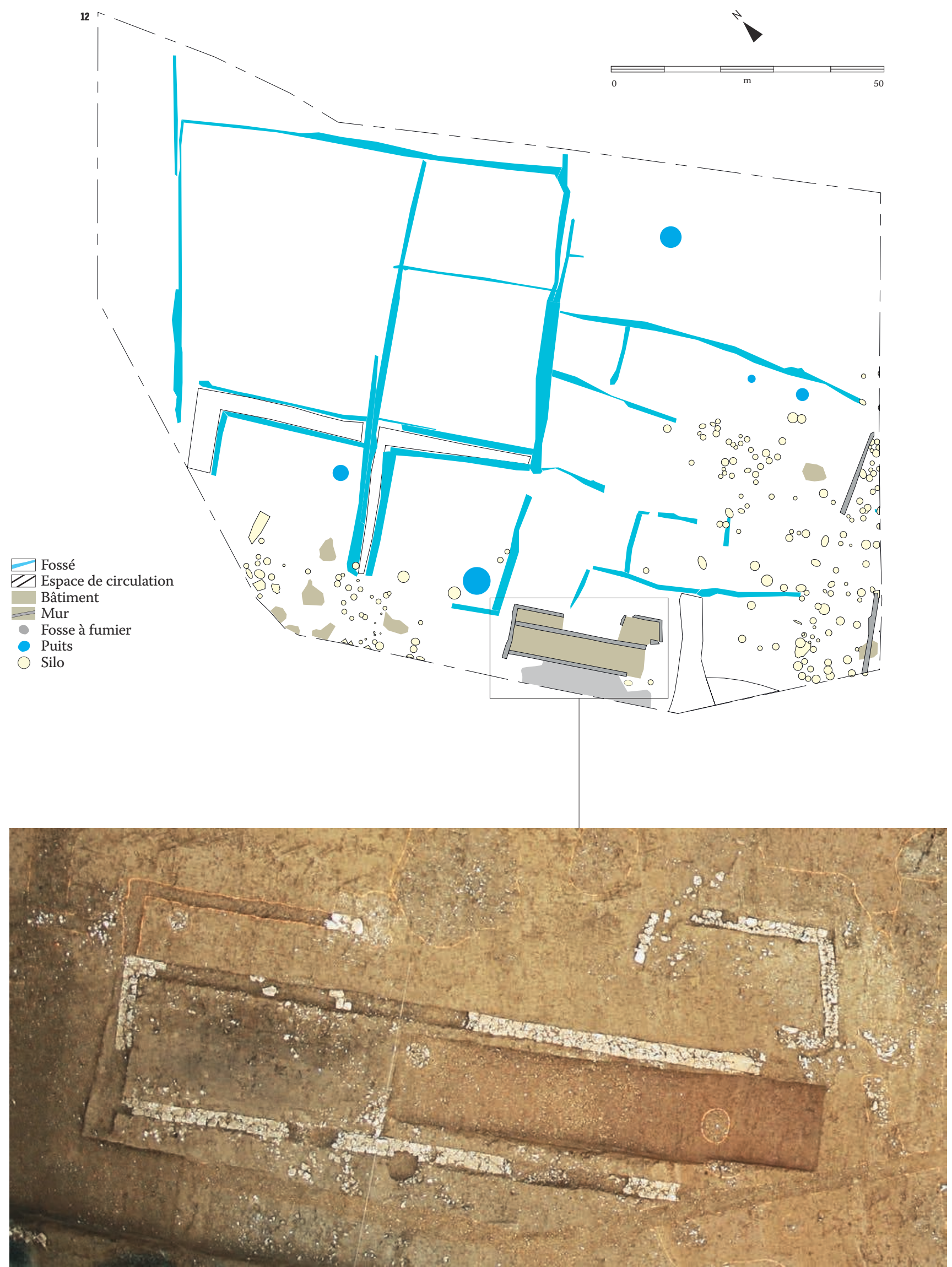


Certes, les toitures du village d'Ugnac au XII ${ }^{\mathrm{e}}$ siècle sont couvertes de tuiles canal, comme l'atteste le four qui les produisait in situ, mais ailleurs, son emploi apparaît plus limité, sans doute concurrencé par la sagne ou le chaume. Le quartier artisanal récemment mis au jour à Castelnau-le-Lez (Hérault) semble confirmer cette préférence pour les toits en végétaux: sur les quelque 69 fours de production de terre cuite mis au jour, seulement 4 , sont de tuiliers, les autres produisant de la vaisselle (travaux en cours de R. Carme/Hadès).

L'espace interne de la maison peut être excavé ; dans ce cas, la bâtisse est installée sur sa propre carrière, un creusement de 0,25 à $0,40 \mathrm{~m}$ de profondeur, qui servira de vide sanitaire. Le matériau extrait est complété par d'autres apports de terre: à Missignac, une zone d'extraction a été ouverte le long d'un chemin d'accès au village. Ici et à La Sabatière, les sols de terre ou de gravier peuvent prendre place directement sur le fond de l'excavation, mais le plancher sur vide sanitaire paraît plus fréquent, en particulier dans les pièces d'habitation. Là, ce sont des indices indirects qui attestent sa présence: les supports des poutres maîtresses, par exemple dans une maison à La Sabatière et à Missignac [ill. 10], ou encore la disposition des aménagements internes [ill. 11].

Dans le cas de creusements internes plus profonds, on parlera de cave. Les sites de Missignac, Lallemand et Saint-Pastour en ont livré des exemples. À Lallemand, un des bâtiments est doté de deux caves de plan ovalaire, contiguës et profondes de 1,20 m. Un plancher devait servir à la fois de plafond bas et de sol du bâtiment [ill. 9]. À SaintPastour, le bâtiment sur cave dispose d'un sous-sol également ovalaire, estimé à 1,30 m de profondeur, dont les parois conservent, une vingtaine de centimètres au-dessus du fond, les cavités d'ancrage des solives d'un sol de bois suspendu. Cette cave disposait donc de son propre plancher sur vide sanitaire. Enfin, la réserve pouvait prendre la forme d'un silo, comme une de celles de Missignac [ill. 11].

On peut enfin s'interroger sur l'existence de greniers, espaces aménagés au premier étage des maisons. Une marche et un emmarchement conservés dans deux maisons de Missignac évoquent la présence d'un étage. Les pièces du rez-de-chaussée pouvaient être dédiées à l'habitation ou aux animaux et l'étage à un grenier ou fenil. La fonction des maisons ou de certaines pièces peut donc être abordée par l'analyse de lorganisation spatiale. Contribuant à cette analyse, l'aménagement de foyers et de cheminées peut déterminer si la pièce est vouée à l'habitat. Les foyers sont entretenus dans des fosses creusées dans le sol à La Sabatière au tournant des VIII ${ }^{\mathrm{e}}$ $\mathrm{IX}^{\mathrm{e}}$ siècles. Plus tardivement, aux $\mathrm{X}^{\mathrm{e}}-\mathrm{XI}^{\mathrm{e}}$ siècles, on connaît, sur ce même site, un exemple de cheminée encastrée dans le mur d'une maison en pierre. À Missignac, peu de foyers sont conservés dans les bâtisses, peut-être parce qu'ils ont disparu avec les planchers, qu'ils aient été au sol ou sur paillasse.
Ici aussi, une cheminée a été mise au jour, également encastrée dans le mur en terre crue, probablement banchée, d'une demeure. L'âtre est simplement formé par une niche aménagée dans le mur, sans renfort d'un parement de pierre, et il s'est rubéfié à l'usage. La sole, initialement constituée d'une pierre plate bien taillée, a été restaurée à deux reprises, en terre puis de nouveau en pierre. Enfin, la cuisson se pratique aussi dans des fours qui sont, en revanche, toujours à l'extérieur, dans les espaces de cour, de place ou à l'angle des rues [ill. 4].

Couvrant une cinquantaine de mètres carrés, dotées de planchers sur vide sanitaire dans les pièces d'habitation et, pour certaines, de caves, celliers, grenier ou fenils et disposant d'un feu, les maisons villageoises, toutes simples qu'elles soient avec leur usage opportuniste de matériaux locaux, n'en paraissent pas moins salubres et bien aménagées.

\section{Une économie principalement agricole}

La vie économique des villages est largement tournée vers l'exploitation du terroir. Les vastes aires d'ensilage soulignent l'importance de la production céréalière, culture dont le rendement est sans doute assez avantageux dans les plaines du Midi où elle a pu être, avec la vigne, une spécialité. Léconomie n'est cependant pas mono-productrice. Le maraîchage se pratique certainement dans les jardins (hortus) que les textes signalent dans les villae ou limitrophes de celles-ci. Lélevage et la consommation des bovins, ovicaprins et porcins sont attestés partout, aux côtés d'une basse-cour de gallinacés (Forest, Olive, 2006). La chasse complète épisodiquement l'approvisionnement de la table, la pêche bien plus régulièrement. À Missignac, on consomme du poisson prélevé presque exclusivement en rivière, en dépit de la relative proximité de la mer. L'outillage, enfin, confirme ces pratiques agricoles (reilles d'araire, faucilles, bêche, mesure à grain [ill. 13,14]) ainsi que la transformation des matières premières: meules pour la mouture des grains ou la presse des olives, peignes à carder, fusaïoles, broches et pesons pour le travail de la laine. On retrouve fréquemment des scories de fer qui témoignent d'une pratique occasionnelle du forgeage, limitée probablement à l'entretien des outils et à la fabrication de la quincaillerie ordinaire. Si les villageois du haut Moyen Âge paraissent ainsi pouvoir vivre en autarcie, d'autres sources attestent leur contribution, directe ou indirecte, à des échanges de moyenne et longue distance. Ainsi, par exemple, le travail du métal se limite t-il aux derniers gestes de la chaîne opératoire, ce qui suppose un approvisionnement en matière première depuis les Cévennes, lieu de production le plus proche. Le commerce se pratique aussi sur de plus longues distances: amphores, vaisselles, lampes et pots en pierre ollaire témoignent de la persistance des échanges avec l'Afrique du Nord, les Alpes ou encore la Bourgogne jusqu'au viri ${ }^{\mathrm{e}}$ siècle. Plus tard, $\mathrm{du} \mathrm{IX}^{\mathrm{e}}$ au XII ${ }^{\mathrm{e}}$ siècle, les monnaies attestent 
les échanges avec l'Anjou, l'Italie et l'Espagne.

À Lallemand, la fouille a mis en évidence une économie pastorale. Aux $\mathrm{X}^{\mathrm{e}}-\mathrm{XI}^{\mathrm{e}}$ siècles, une partie de l'aire d'ensilage est abandonnée au profit d'une bergerie dotée d'enclos de pâture ou de parcage [ill. 13].

La bergerie présente un plan rectangulaire de 22,10 m de long par 5,45 m de large hors œuvre, soit une surface intérieure de $90 \mathrm{~m}^{2}$. Compte tenu de l'arasement des structures, il est difficile de restituer la nature des élévations : pierre? terre crue? terre et bois? L'existence d'un fenil à l'étage peut être envisagée. La nature des couvertures nous échappe, mais l'absence de tuiles dans les niveaux de démolition plaide en faveur d'une toiture végétale. Les sols ont disparu: seul subsiste, à l'intérieur du bâtiment, un probable radier. Sur sa façade orientale, le bâtiment est doté d'un appentis rectangulaire ouvert sur le sud. Accolée à cette même façade, une exèdre de plan trapézoïdal, très arasée, présente une surface interne de $15 \mathrm{~m}^{2}$ environ. Une vaste fosse destinée au recueil des déchets et effluents est accolée à la façade occidentale.

Par ses dimensions et son orientation, le bâtiment évoque les bergeries étudiées sur la plaine de la Crau (Badan et al., 1995). Par son association avec une fosse à effluents, il est à rapprocher aussi de la bergerie du Mas de Fourques à Lunel (Ott, 2009). Tous ces éléments de comparaison sont antiques et les exemples médiévaux font défaut dans la région, mais les formes de ce type d'architecture vernaculaire varient peu entre l'Antiquité et le XIX ${ }^{\mathrm{e}}$ siècle. Dans les bergeries modernes, on compte trois brebis au mètre carré. Même s'il faut se garder de transposer les données récentes aux pratiques médiévales, on peut estimer le cheptel de Lallemand entre 250 et 300 têtes.

Un vaste système parcellaire contemporain se développe au nord-est du bâtiment. Les enclos fossoyés qui le constituent couvrent des espaces allant de $100 \mathrm{~m}^{2}$ à $1500 \mathrm{~m}^{2}$, les parcelles les plus petites étant situées à proximité de la bergerie. Ces parcelles communiquent au moyen de ponceaux ou de couloirs permettant le tri des bêtes, leur traite ou leur tonte. Des espaces voués à la circulation des hommes et des bêtes sont identifiés au sud et au nord de la bergerie.

Dans l'attente de l'étude archéozoologique, on ignore si l'abattage et la boucherie se pratiquaient sur place et si les bêtes en excédent étaient vendues sur pied. Aucun ustensile lié à la fabrication du fromage n'a été retrouvé, sans doute parce que les faisselles étaient en bois ou en vannerie. En revanche, un grand nombre de pesons en terre cuite a été retrouvé sur le site et atteste le travail de la laine. Les outils liés à la transformation de la laine - fusaïoles, broches, peigne à carder [ill. 15, 16] se rencontrent également sur la plupart des établissements du Languedoc, notamment à Missignac et à Saint-Pastour.
Ainsi, à Lallemand, serions-nous en présence d'une exploitation polyvalente de l'ovicaprin: production de viande, de lait et de produits laitiers, de laine et de lainages, mais aussi sans doute de peaux et de fumier. Les questions relatives à ces productions sont encore nombreuses en Languedoc (Forest, Rodet-Belarbi, 2009): quelle était la part respective des différentes espèces? Avons-nous affaire à la gestion familiale d'un troupeau privé ? L'activité est-elle au contraire gérée par la collectivité? Les études historiques, archéologiques et archéozoologiques actuellement en cours sur les établissements découverts récemment apporteront un éclairage nouveau.

\section{Une restitution en devenir}

Les investigations archéologiques de ces trois dernières années ont levé le voile sur un pan de l'histoire languedocienne en apportant une documentation abondante sur les premiers temps du village médiéval. Les traits qui s'en dégagent et que les études en cours viendront progressivement préciser, renouvellent d'ores et déjà l'image négative véhiculée jusqu'ici. Il apparaît que le villageois du haut Moyen âge habitait une maison aménagée avec soin, disposait, en propre ou en partage, d'équipements sociaux (l'église, le cimetière), économiques puis défensifs qui assuraient son accompagnement spirituel, sa subsistance et sa sécurité. Il participait directement ou indirectement au commerce interrégional et plus lointain. Bientôt, les résultats des études biologiques des habitants de ces villages, dont les tombes et les squelettes ont été retrouvés, éclaireront aussi leur santé et permettront de mesurer, particulièrement, la récurrence des pénuries alimentaires auxquelles la population a pu être soumise. 
13. Deux faucilles de

Missignac, des $\mathrm{vII}^{\mathrm{e}} \mathrm{x}^{\mathrm{e}} \mathrm{s}$.

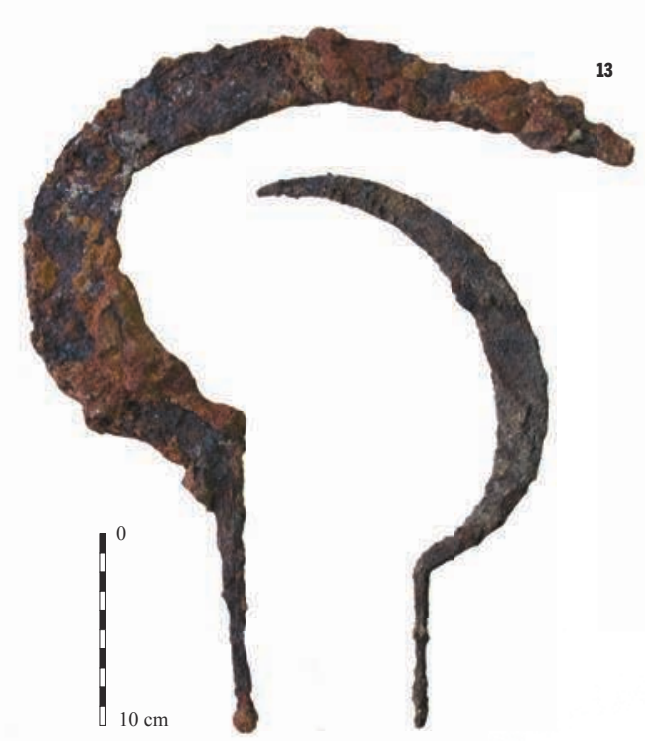

15

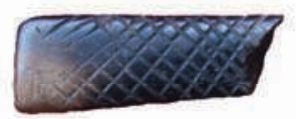

14

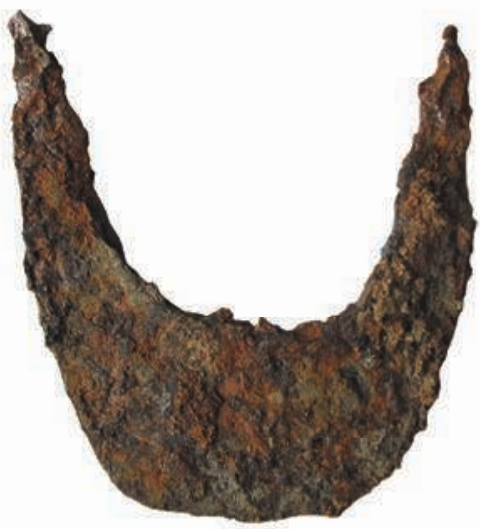

$0 \quad 10 \mathrm{~cm}$ (à gauche) et des $\mathrm{Xl}^{\mathrm{e}}-\mathrm{xII}^{\mathrm{e}} \mathrm{s}$.

(à droite).

14. Bêche en fer

de Saint-Pastour.

15. Broches de tissage en

os retrouvées à Lallemand

et Saint Pastour.

16. Peigne à carder

en fer retrouvé dans

le comblement d'un silo

de Saint-Pastour.
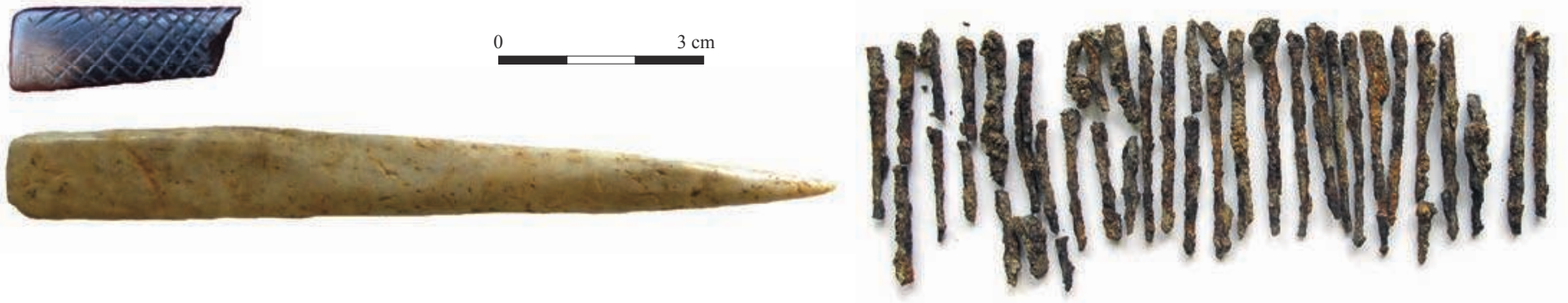

\section{Références bibliographiques}

BADAN O., Brun J.-P., Conges G., 1995, «Les bergeries romaines de la Crau d'Arles: les origines de la transhumance en Provence », Gallia, 52, p. 263-310.

Bourin M., Durand R., 1984, Vivre au village au Moyen Âge: les solidarités paysannes $d u X I^{e}$ au XIII ${ }^{e}$ siècle, Paris, Messidor/Temps Actuels (coll. La passion de l'Histoire), $258 \mathrm{p}$.

Faure-Boucharlat E. (DIR.), 2001, Vivre à la campagne au Moyen Âge : l'habitat rural du $v^{e} a u$ XII ${ }^{e}$ s. (Bresse, Lyonnais, Dauphiné) d'après les données archéologiques, Lyon, Alpara (coll. Documents d'archéologie en Rhône-AlpesAuvergne, 21), $429 \mathrm{p}$.

Forest V., Olive C., 2006, Études archéozoologiques, in Maufras O. (DIR.), Habitats, nécropoles et paysages dans la moyenne et la basse vallée du Rhône (XII $-X V^{e} s$.): contribution des travaux $d u$ TGV-Méditerranée à l'étude des sociétés rurales médiévales, Paris, Éd. de la Maison des sciences de l'homme (coll. Daf, 98), p. 241-261.

Forest V., Rodet-Belarbi I., 2009, «Viandes animales dans le Languedoc-Roussillon rural médiéval: bilan 2010 ", Ruralia, VIII, p. 91-112.

Garnier B., Garnotel A., Mercier C., Raynaud C., 1995, «De la ferme au village, Dassargues du $v^{e}$ au $X I I^{\mathrm{e}}$ s. (Lunel, Hérault) », Archéologie du Midi médiéval, 13, p.1-78.
Gentili F., 2010, «L'organisation spatiale des habitats ruraux du haut Moyen Âge: l'apport des grandes fouilles préventives. Deux ensembles franciliens: Serris "les Ruelles" (Seine-et-Marne) et Villiers-le-Sec (Val-d'Oise)", in CHApelot J. (DIR.), Trente ans d'archéologie médiévale en France: un bilan pour un avenir, Caen, Publications du CRAHM, p.119-131.

Jandot C., Barberan S., Barbey S., 1999, Saint-Jean d'Aureilhan, Béziers, Hérault, Nîmes/Montpellier, Afan/SRA, 92 p. et annexes non paginées [DFS de fouille archéologique de sauvetage urgent, non publié].

Le Roy L., Berdeaux-Le-Brazidec M.-L., Malignas A., Rovira N., 2011, «De la villa antique à l'établissement alto-médiéval de la Maladrerie à Saillans (Drôme) : permanences et mutations jusqu'au viII ${ }^{\mathrm{e}}$ s. », Archéologie du Midi médiéval, 29, p. 3-42.

MAUfras O., à paraître, «Aperçu des villae médiévales de la Vistrenque à Nîmes (Gard): répartition, formes et héritage antique», in RECHIN F. (DIR.), Actes de la table ronde tenue à Pau en 2012 (Circa Villam).

Maufras O., Mercier C., 2012, «La villa de Codols et les villae de la plaine du Vistre d'après les sources historiques ", in Pomarèdes H., Maufras O., Barberan S., Sauvage L., La villa de Saint-André de Codols (Nîmes, Gard) du I $I^{e r}$ au XII $I^{e}$ s. de n. è, Lattes, CNRS (coll Monographie d'archéologie méditerranéenne, 32), 2012, p. 211-23o.
Oтт M., 2009, «L'exploitation agricole du Mas de Fourques aux I ${ }^{\mathrm{er}}$ et $\mathrm{II}^{\mathrm{e}} \mathrm{s}$. de n. è. (Lunel, Hérault)», Revue archéologique de Narbonnaise, 43, p. 193-205

PISKORZ M., MAUFRAS O., AUDOUIT F., RECOLIN A., RAYNAUD C., 2002, La Jasse d'Isnard à Aimargues (Gard); l'église carolingienne de la villa Telianum, Nîmes/Montpellier, Inrap/SRA, 4o p. [DFS de diagnostic archéologique, non publié].

Pomarèdes H., Maufras O., Barberan S., Sauvage L., 2012, La villa de Saint-André de Codols (Nîmes, Gard) du $I^{r}$ au XII ${ }^{e}$ s. de n. è, Lattes, CNRS (coll Monographie d'archéologie méditerranéenne, 32), $436 \mathrm{p}$.

Pomarèdes H., Maufras O., 2012, « La villa de Codols et le peuplement de l'espace périurbain nîmois », in Pomarèdes H., Maufras O.,

Barberan S., Sauvage L., La villa de Saint-André de Codols (Nîmes, Gard) du Ir au XII $I^{e}$ s. de n. è, Lattes, CNRS (coll Monographie d'archéologie méditerranéenne, 32), 2012, p. 389-402.

SCHNEIDER L., 2006, «Vers la Méditerrannée : un regard sur les grandes aires d'ensilage médiévales », in Maufras O. (DIR.), Habitats, nécropoles et paysages dans la moyenne et la basse vallée $d u$ Rhône $\left(V I I^{e}-X V^{e}\right.$ s.): contribution des travaux $d u$ TGV-Méditerranée à l'étude des sociétés rurales médiévales, Paris, Éd. de la Maison des sciences de l'homme (coll. Daf, 98), p. 440-445.

SCHNEIDER L., 2014, «Les églises rurales de la Gaule ( $\mathrm{V}^{\mathrm{e}}$-VIII ${ }^{\mathrm{e}} \mathrm{s}$.). Les monuments, le lieu et l'habitat: des questions de topographie et d'espace », in GAILlARD M. (DIR.), L'empreinte chrétienne en Gaule du IV $V^{e}$ au IX $X^{e}$ s, Bruxelles, Brepols (coll. «Culture et société médiévales »), p. 419-468. 